\title{
Evaluación de la competencia lectora en infantes, basado en el paradigma interconductual
}

\section{Julio Alejandro Gómez Plascencia, Aidee Jaquelin Ortega Jiménez, Alejandra Rodríguez Solís, Ahtziri Abigail Ávalos Molina, Israel Christian Huerta Solano}

\section{Resumen}

En la actualidad las investigaciones que estudian la lectura han sido justificadas mediante el paradigma cognitivo, siendo a partir de éste que se ha delimitado y evaluado dicha competencia. Ante ello, en este estudio se propone un instrumento de evaluación de la competencia lectora para infantes a partir del paradigma interconductual basado en la propuesta de Kantor y Ribes. Se retoman los cinco niveles funcionales que estructuran el comportamiento e interacciones lectoras, cada uno sugiere un nivel de interacción mayor a su predecesor. El instrumento fue administrado a seis niños en edad escolar correspondiente al segundo grado de primaria. Se concluye que los infantes evaluados tienen habilidades necesarias para realizar niveles de menor nivel de complejidad de interacción (lectura en voz alta, dictado, deletreo), sin embargo, no tienen habilidades necesarias para la realización de niveles de mayor nivel de complejidad de interacción (comparación de texto, expresión de una opinión, conocimiento social. 
Palabras clave: Competencia lectora; interconductismo; evaluación; infantes.

\section{Introducción}

La lectura constituye una competencia importante para el desarrollo psicológico de cualquier persona, considerando que su uso repercute en lo educativo, social y recreacional. De igual manera, la lectura permite la interacción con una lengua, las creencias, costumbres y cultura de la persona (Palomino, 2011).

A partir de la importancia que tiene la adquisición de la competencia lectora, la psicología ha tenido un papel importante en la producción de conocimiento respecto a dicho tópico, lo que ha llevado a la elaboración y administración de instrumentos con este propósito (Solé, 2001).

Consecuentemente, es necesario señalar que la generación de un instrumento no sólo se encuentra usualmente mediada por el análisis estadístico, ya que "se puede considerar que la elaboración y/o aplicación de instrumentos para medir y evaluar el aprendizaje se encuentra necesariamente relacionada con la teoría que les subyace, como a los constructos o variables que el especialista pretende medir" (Bazán, Castañeda, Macotela y López, 2004: 845). Es decir, se hace necesaria una delimitación de lo que se quiere cuantificar a partir de un paradigma que permita comprender las propiedades del fenómeno estudiado.

En la actualidad, hay una cantidad considerable de estudios dedicados a evaluar la lectura, sobre todo bajo la perspectiva teórica cognitiva, basándose en el estudio de la mente, en términos de procesamiento de la información y entendiendo el aprendizaje como la adquisición de conocimiento (Cabrera-Cortés, 2003; Pinker, 2003). Así, a partir del paradigma referido se han desarrollado distintos modelos explicativos de la lectura, entre éstos el de procesamiento ascendente y descendente, de interacción y el de la doble ruta (Alonso y Del Mar, 1985; Ferreres, Martínez, Jacubovich, Olmedo y López, 2003), entre otros.

Sin embargo, pareciera que, sin importar el modelo utilizado, la lectura en el paradigma cognitivo se entiende como el procesamiento de información en el que es necesaria la coor- 
dinación de procesos automáticos, no conscientes, que se encuentran en la mente del individuo para decodificar los símbolos y la comprensión de éstos, a lo que se suma la necesidad de usar estrategias cognitivas tales como la memoria de trabajo, el monitoreo, la memoria fonológica y la teoría de la mente para la realización de la lectura (Zorrilla, 2005).

Si bien es cierto que lo anterior ha contribuido al estudio de la competencia lectora, como lo demuestra el desarrollo de distintos instrumentos a partir del paradigma cognitivo, entre éstos el subtest de procesos semánticos del test PROLEC (Cuetos, Rodríguez y Ruano, 1996), la Prueba de Comprensión Lectora de Lázaro, (1980 y 1996), las Subpruebas de Lectura y Escritura de la Batería Neuropsicologíca (ENI) (Matute, Rosselli, Ardila y Ostrosky-Solis, 2005). Retomando lo expuesto por Thomas Kuhn (1961 y 2004), se debe señalar que la investigación en una comunidad científica se encuentra ligada a un paradigma dominante, lo que determina el corpus teórico para el abordaje explicativo de ciertos fenómenos, por lo tanto, podemos considerar que la delimitación teórica actual del constructo "lectura" ha sido justificado de sobremanera bajo el dominio del paradigma cognitivo.

Contrariamente, dicho dominio conceptual y práctico deja de lado fenómenos y anomalías que no pueden ser explicados bajo los postulados mentalistas. Es decir, la delimitación de constructos desde el citado paradigma, al dejar de lado fenómenos que no se pueden analizar desde sus postulados, ha servido para delimitar la investigación sobre la lectura, repercutiendo a su vez en la evaluación de dicha competencia. Reiterando lo expuesto por Kuhn, respecto al periodo de crisis en un paradigma, es necesario el desarrollo teórico y práctico de un paradigma diferente que permita trabajar sobre aquellos fenómenos que el anterior no pudo resolver, o considerar el mismo fenómeno mediante un nuevo corpus teórico que permita entender y evaluar de manera diferente el fenómeno de interés, eliminando las restricciones de la investigación científica producidas por el paradigma anterior. 


\section{Lectura desde el cognitivismo}

De acuerdo con el paradigma cognitivo, la lectura es un proceso que se facilita conforme las competencias verbales que el infante haya adquirido en su "etapa preescolar". En otras palabras, dicho proceso se da a la par del desarrollo del lenguaje oral y escrito, lo que tendrá efecto en el aprendizaje básico de la lectura. Así, por medio del dominio del lenguaje oral, la evolución de procesos cognitivos, que son fundamentales para el desarrollo de la lectura, fungirán en la configuración de procesos de mayor complejidad que se verán reflejados en la lectura de comprensión, no obstante, es necesario recordar que la lectura se ha articulado a partir del desarrollo de habilidades psicolingüísticas previas, puesto que no sólo surge como un proceso de apropiación cultural del ambiente escolar (Bravo, 2013).

En un sentido similar, Whitehurst y Lonigan (1998, citados en Bravo, 2013) describen dos conjuntos de destrezas y procesos que surgen en el aprendizaje de la lectura que se relacionan entre sí, aunados al dominio externo durante la acción de leer, tales como el lenguaje oral, la conciencia alfabética y el conocimiento de los textos, además del dominio interno o neuropsicológico cognitivo (la conciencia fonológica, la velocidad de nominación y la memoria verbal operacional).

Cabe indicar que en el paradigma cognitivo, la evolución del proceso psicológico en el aprendizaje de la lectura se desarrolla desde la conciencia del lenguaje hasta el dominio de la decodificación y reconocimiento de las palabras de manera visual, lo que se explica por medio del concepto de emergencia de la lectura y de la escritura. Consecuentemente, la emergencia lectora será entendida como "el desarrollo de procesos psicolingüísticos que se adquieren de manera temprana, siendo necesarios para el aprendizaje lector, aludiendo a la conciencia fonológica, semántica, sintáctica, alfabética, así como el conocimiento de palabras y la memoria verbal" (Bravo, 2013).

\section{Acerca del paradigma interconductual kantoriano}


El paradigma interconductual surge en los años veinte con la propuesta de Kantor y Smith (1975 y 2015) como alternativa a las perspectivas dominantes en psicología, misma que desde un inicio se distanció de las propuestas mentalistas y que tomó como su objeto de estudio la interconducta, entendida como la interacción entre el organismo y su ambiente.

A diferencia del conductismo, que tiene un esquema diacrónico ( $E \quad R)$, en el que se tiene un solo estímulo y una sola respuesta, el paradigma interconductual alude al concepto de campo en el que se presentan eventos psicológicos mediante interrelaciones sincrónicas $(E \quad r)$ no lineales, por lo que para el estudio de dichas relaciones se hace uso del constructo de campo interconductual, que se refiere a la interacción psicológica en la que se reconoce que el organismo actúa en relación con objetos, eventos y otros organismos que, a su vez, actúan sobre el organismo, de tal manera que el interés analítico no se concentra en respuesta y estímulo, sino en su interacción en la que una y otro desarrollan funciones específicas. Dicho de otra forma, el interés analítico se concentra en la interacción de funciones de estímulo (FE) y funciones de respuesta (FR) pero no en alguna de ellas de modo independiente.

\section{La lectura desde el modelo interconductual}

Es necesario conocer que la conducta lingüística es un acto comunicativo que implica una relación de tres elementos: un referidor (quien habla, seña, escribe o dibuja), un referido (quien escucha, lee u observa) y un referente (de quien se habla, seña, escribe o dibuja); es decir un evento por el cual se comunica algo a alguien.

Por tanto, la conducta lingüística corresponde a una interacción biestimulacional, en la que uno de los objetos de estímulo con los que se interactúa corresponde a la persona a la que se le habla, el texto que se escribe, la persona a la que se le hacen señas o la figura que se dibuja, y el segundo objeto estímulo es aquello de lo que se habla, escribe, gesticula o dibuja (Varela, 2008; Kantor y Smith, 1975 y 2015). Obsérvese entonces que el texto pone en contacto al lector con un evento de referencia al 
igual que el habla pone en contacto al referidor con un evento de referencia que es presentado en forma oral (Kantor y Smith, 1975 y 2015).

En otras palabras, la interacción biestimulacional requiere para su efectiva ejecución la competencia adecuada en el sujeto y así lograr una interacción eficiente para o con algo a través del segmento interconductual, entendiendo por competencia "la aptitud o idoneidad para hacer algo" (Varela, 2008). Así pues, es necesaria "la adquisición de ciertas competencias dependiendo de la actividad que se pretende clasificar, de los instrumentos que se emplean para su realización, así como de los elementos que se involucran, como lo es el conocimiento de la tarea" (Varela, 2008: 28).

Ribes y López (1985) identificaron cinco tipos de interacciones que se diferencian por la complejidad que requiere la realización de una tarea dentro de la conducta lingüística a saber contextual, suplementaria, selector, sustitutiva referencial y sustitutiva no referencial, con base en el criterio de mediación, entendido como el proceso mediante el cual un elemento del campo interactivo se torna crítico en la estructuración del conjunto de relaciones entre los distintos factores del campo. Por otro lado, el desligamiento funcional es explicado como el grado de autonomía relativa que el comportamiento de los individuos puede mostrar al responder respecto de las propiedades físico-químicas, espacio-temporales de la situación en que la conducta tiene lugar y convencionales.

Así, a partir de esta taxonomía de la interconducta propuesta por Ribes y López (1985), Trigo, Martínez y Moreno (1988, citado en Bazán-Ramírez, Urbina-Pérez, Domínguez-Márquez, Mansillas-Cervantes y Gómez-Manjarrez, 2011) plantean que estos cinco niveles de interacción referidos se estructuran en tres niveles generales de desligamiento funcional:

1. Desligamiento intrasituacional: Este nivel se caracteriza por una correspondencia directa entre el individuo y un evento concreto con el cual interactúa, éste puede ser personas u objetos que se encuentran dentro de la situación o episodio interactivo; la interacción es intrasituacional, es decir se mantie- 
nen ligadas estas relaciones concretas situacionales. En lo que corresponde a la lectura, se "demanda la correspondencia morfológica entre la actividad del lector y las propiedades físicas del texto" (Morales-Chávez, Alemán-Baños, Canales-Sánchez, Arroyo-Hernández y Carpio-Ramírez, 2013: 81).

Además, las interacciones implican actividades que son demandadas por elementos que se encuentran en el texto. En este nivel se dan tres tipos de interacción: a) Contextual: en la que se tiene una reacción al contexto, sin introducir cambios en éste. Se tiene como ejemplo, en referencia con la competencia lectora, la lectura en voz alta; b) Suplementaria: en ésta se tiene una participación de respuesta del individuo que afecta la estructura del texto; y c) Selectora: en ésta no sólo la acción del individuo afecta a la situación, sino que dicha acción responde dependiendo de diferentes estímulos. Un ejemplo es la selección de una opción pertinente a lo que demanda un texto (Morales-Chávez et al., 2013).

2. Desligamiento extrasituacional: En este nivel la conducta implícita en la correspondencia interactiva es independiente en relaciones las propiedades situacionales, es decir desligada del aquí y ahora de los objetos y eventos relacionados; el individuo sustituye una situación o le atribuye funciones no aparentes. En lo referente a la lectura, se presenta una correspondencia entre las acciones del lector con los elementos presentes del texto, pero es necesario el desarrollo de relaciones lingüísticas entre elementos referidos en el texto y los elementos de otras situaciones específicas. En este nivel se da la interacción sustitutiva referencial en la que "se establece una correspondencia entre elementos del texto y las acciones que el lector debe desarrollar en una situación 'extra-texto'" (Morales-Chávez et al., 2013: 81). Es decir, las acciones del individuo ya no responden a elementos del texto presentes en el aquí y ahora, 